\title{
Porous Carbon Nanofiber-Modified Carbon-Fiber Microelectrodes for Dopamine Detection
}

\section{Supporting Information}

Blaise J. Ostertag ${ }^{1}$, Michael T. Cryan ${ }^{1}$, Joel M. Serrano ${ }^{2}$, Guoliang Liü, and Ashley E. Ross ${ }^{1{ }^{*}}$

'University of Cincinnati

Department of Chemistry

312 College Dr.

404 Crosley Tower

Cincinnati, OH 45221-0172

Office Phone \#: 513-556-9314

Email: Ashley.ross@uc.edu

*Corresponding author

${ }^{2}$ Virginia Polytechnic Institute and State University

Department of Chemistry, Macromolecules Innovation Institute, Division of Nanoscience, Academy of Integrated Science

800 West Campus Dr.

Blacksburg, VA, 24061

\section{Table of Contents}

S 2: Abstract of included materials

S 3-4: Supporting methods

S 5: $\mathrm{CO}_{2}$ and $\mathrm{N}_{2}$ physisorption isotherms for pore size analysis fo PCF

S 6: Energy Dispersive Spectroscopy (EDS) of synthesized PCF

S 7: Dip coating modification analysis of PCF-CF

S 8: Drop casting modification analysis of PCF-CF

S 9: Current versus time trace of bare CF and PCF modified CF

S 10: Electrodeposition control experiment using only dimethyl formamide (DMF)

S 11: Analysis of bare CF and PCF modified CF at various frequencies

S 12: Current versus $v^{1 / 2}$ for $5 \mathrm{mM} \mathrm{Ru}\left(\mathrm{NH}_{3}\right)_{6}+2 /+3$ of bare $\mathrm{CF}$ and PCF modified

S 13: Electrochemical performance analysis of PCF, GO-mod. CFME, and CNT Yarns

S 14: Supporting References 


\section{Abstract of included materials:}

Here, we present an effective method for modifying carbon-fiber microelectrodes (CFME) using porous carbon nanofibers (PCFs) for improved dopamine detection using fast-scan cyclic voltammetry (FSCV). This Supporting Information document contains both Supporting Methods and Supporting Figures for the manuscript. Supporting Methods consist of reagents, synthetic procedures, and characterization used to fabricate the porous carbon nanofibers. Supporting Figures consist of a higher magnification SEM image of PCF nanofibers, dopamine CV, and background current analysis for the deposition methods of dip coating and drop casting, and a control experiment in which dopamine CV currents were analyzed before and after electrodeposition of $\mathrm{N}, \mathrm{N}$-dimethylformamide (DMF) scanning from -1.2 to $1.5 \mathrm{~V}$ at $1 \mathrm{HZ}$ and $5 \mathrm{~V} / \mathrm{s}$. A table is shown comparing other carbon-based material modifications onto carbon-fiber microelectrodes with FSCV. 


\section{Supporting Methods}

\section{Chemicals for PCF Synthesis}

Methyl methacrylate (MMA, $\geq 99 \%$ ), acrylonitrile (AN, $\geq 99 \%$ ), 2,2'-azobis(2methylpropionitrile) (AIBN $\geq 98 \%$ ), benzene ( $\geq 99 \%$ ), aluminium oxide (activated, neutral, Brockmann Activity I), N,N-dimethylformamide (DMF, $\geqslant 99.7 \%$ ), and dimethyl sulfoxide (DMSO, $\geqslant 99.9 \%$ ) were purchased from Sigma-Aldrich. Cumyl dithiobenzoate (CDB) was synthesized via a previously reported method. ${ }^{1}$ Pumped methanol was used for polymer precipitation. MMA and AN were purified by alumina column filtration to remove inhibitors. Polymer Synthesis

Poly(methyl methacrylate)-block-poly(acrylonitrile) (PMMA-b-PAN) was synthesized via reversible addition-fragmentation chain transfer (RAFT) polymerization according to a previous report with modifications. ${ }^{2,3}$ First, a mixture of MMA $(70.0 \mathrm{~mL}, 618$ $\mathrm{mmol}$, CDB (169 mg, $0.318 \mathrm{mmol}$ ), and AIBN (50.8 mg, $0.310 \mathrm{mmol}$ ) were dissolved in benzene $(110 \mathrm{~mL})$ in a $500-\mathrm{mL}$ Schlenk flask. The solution was subjected to three cycles of freeze-pump-thaw (FPT) followed by back-filling with $\mathrm{N}_{2}$ to remove dissolved oxygen gas. The reaction proceeded at $60^{\circ} \mathrm{C}$ for $48 \mathrm{~h}$ resulting in a PMMA macro-chain transfer agent (CTA). The PMMA macro-CTAs were precipitated in methanol, filtered, and dried under vacuum for $16 \mathrm{~h}$.

The purified PMMA macro-CTAs were then used to synthesize PMMA- $b$-PAN block copolymer (BCP). BCP was synthesized by the mixture of PMMA macro-CTA (6.50 g, 110. umol), AN (26.0 mL, $433 \mathrm{mmol})$, AIBN (4.4 mg, $27 \mu \mathrm{mol})$, and DMSO (72.2 mL). The AIBN was added last to the mixture immediately preceding the three FPT cycles. The mixture was then heated in an oil bath at $65^{\circ} \mathrm{C}$ under $\mathrm{N}_{2}$ atmosphere for $10 \mathrm{~h}$ which resulted in precursors with number-average molecular weights: PMMA (80 kDa) and PAN (40 kDa). The PMMA- $b$-PAN block copolymer was purified following the same steps as the PMMA 
macro-CTAs. The mixture was then diluted with $80 \mathrm{~mL}$ of DMF and then precipitated into methanol. The precipitates were collected by filtration, washed with methanol. The number average molecular weights were measured with size exclusion chromatography (SEC) (EcoSEC HLC-8320GPC) and analysed with an attached light scattering detector (DynaPro Nanostar photometer).

\section{Synthesis and electrospinning porous carbon nanofibers}

The block copolymers were then dissolved in DMF (16 wt. \%) overnight ( 12 h). The dissolved BCPs were electrospun at a flow rate of $0.030 \mathrm{~mL} \mathrm{~min}^{-1}$ with $17 \mathrm{kV}$ (using high power supply, Model P030HP1, Acopian Technical Company) and collected onto an aluminium foil coated drum with a spin rate of $\sim 140 \mathrm{rpm}$. The as-spun fibers were oxidized at $280^{\circ} \mathrm{C}$ with a heating rate of $1^{\circ} \mathrm{C} \mathrm{min}-1$ for $8 \mathrm{~h}$ under air to stabilize the fibers. Afterwards the fibers were then pyrolyzed at $800{ }^{\circ} \mathrm{C}$ for $1 \mathrm{~h}$ at a heating rate of $10^{\circ} \mathrm{C} \mathrm{min}^{-1}$ in a tube furnace (Model STF55433C-1, Lindberg/Blue M) under $\mathrm{N}_{2}$ atmosphere $\left(\mathrm{N}_{2}\right.$ flow rate, 200 standard cubic centimetres per minute) which resulted in porous carbon fibers (PCF).

\section{Surface Characterization of PCF}

$\mathrm{N}_{2}$ and $\mathrm{CO}_{2}$ physisorption were performed at 77 and $273 \mathrm{~K}$, respectively, using a 3Flex pore analyzer (Micrometric Instruments Company). Prior to physisorption, PCF were degassed at $90^{\circ} \mathrm{C}$ for $1 \mathrm{~h}$ and $300^{\circ} \mathrm{C}$ for $10 \mathrm{~h}$ to remove any surface adsorbents. Pore width distributions were calculated using non-local density functional theory (NLDFT). 


\section{Supporting Figures and Discussion}

A.

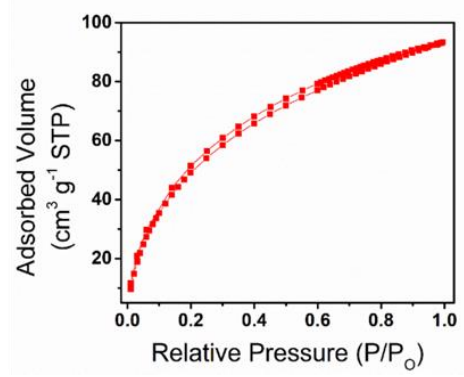

B.

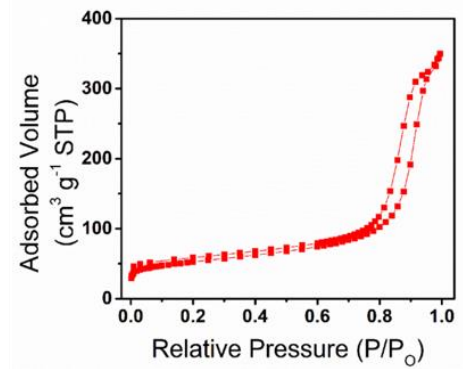

C.

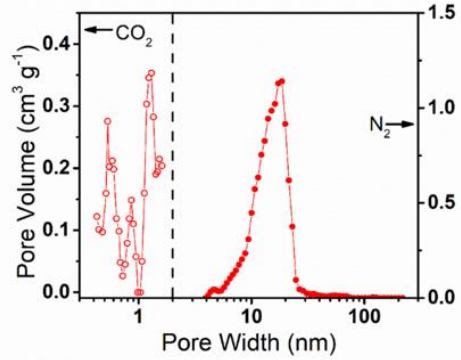

Figure S-1: $\mathrm{CO}_{2}$ and $\mathrm{N}_{2}$ physisorption isotherms illustrate microporous and mesoporous PCF framework with uniform, controlled pore width distributions. (A) $\mathrm{CO}_{2}$ physisorption isotherm. (B) $\mathrm{N}_{2}$ physisorption isotherm. (C) Plot of pore width distributions across PCF framework.

The pore sizes and surface areas of the porous carbon fibers (PCF) were determined by gas physisorption. $\mathrm{CO}_{2}$ and $\mathrm{N}_{2}$ adsorption-desorption isotherms revealed type I and IV isotherms (Figure S-2, A-B), respectively. ${ }^{1-3} \mathrm{CO}_{2}$ physisorption probes PCF micropores and, utilizing nonlocal density functional theory (NLDFT), the micropore size distribution was calculated (Figure S2, C). The micropore size distribution, as determined from $\mathrm{CO}_{2}$ adsorption, exhibited multiple distinct peaks centered at $\sim 0.53,0.86$, and $1.29 \mathrm{~nm}$.

The $\mathrm{N}_{2}$ isotherm showed a hysteresis between $0.7<P / P_{0}<0.9$ which indicated mesopores within the carbon fibers (Figure S-2, B). ${ }^{4,5}$ The mesopore size distribution was also calculated by NLDFT with $\mathrm{N}_{2}$ physisorption showing a unimodal peak at $\sim 18 \mathrm{~nm}$ owing to the well-controlled microphase-separation of PMMA- $b$-PAN. The PMMA domains serve as a sacrificial block which fully decomposes during pyrolysis and leads to the developed mesopores. These pore sizes and distributions strongly depend on the block copolymer molecular weight and composition. ${ }^{6}$ 


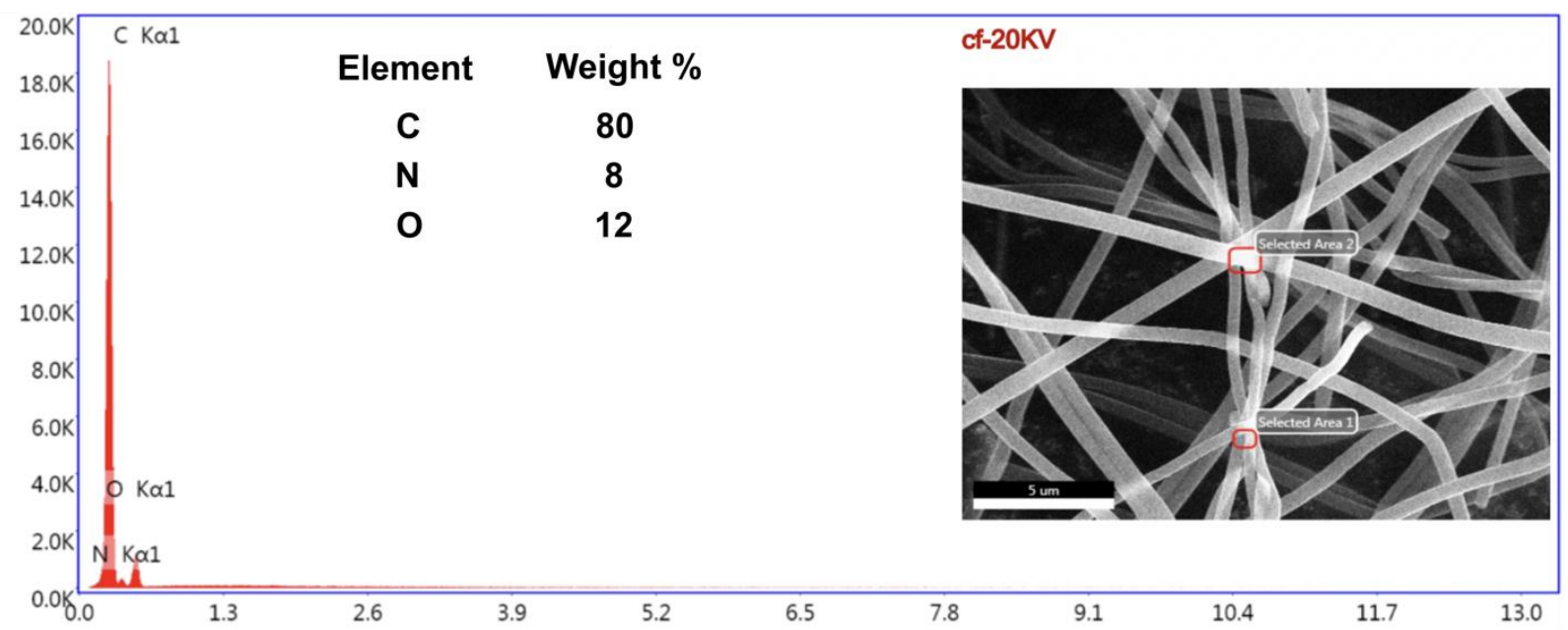

Figure S-2: Energy dispersive spectroscopy (EDS) of PCF material. EDS measurements demonstrates the presence of carbon, nitrogen and oxygen on the surface of the pyrolyzed PCF material, as expected. 
A

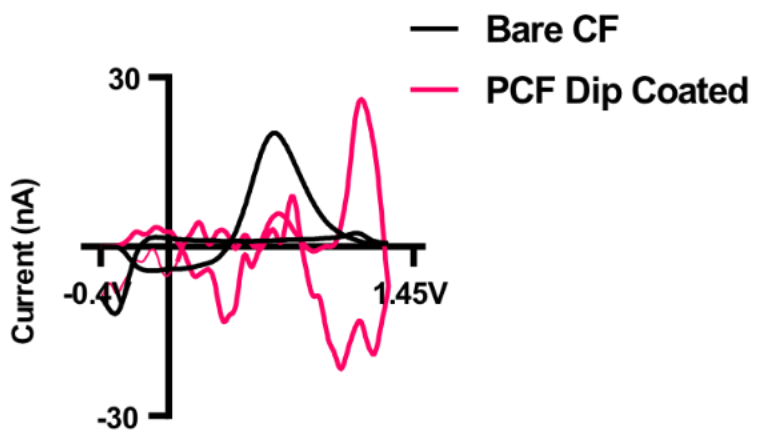

B

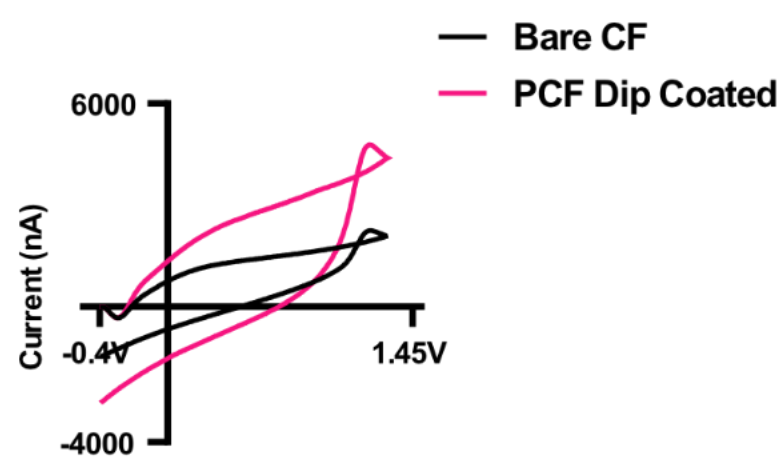

C.

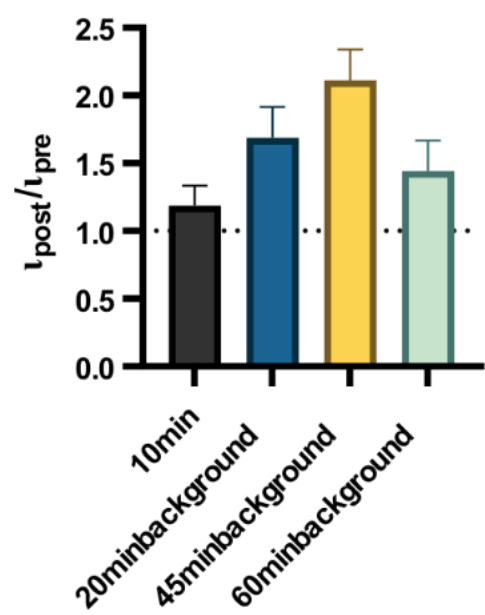

Figure S-3: Modifying CFME with porous carbon nanofibers using dip coating shows major increases in background current accompanied by decreased signal-to-noise. (A) Example CV of $1 \mu \mathrm{M}$ DA before (black) and after (pink) dip coating $1 \mathrm{mg} / \mathrm{mL}$ PCF/DMF for 45 minutes. (B) Example background current $\mathrm{CV}$ before and after dip coating $1 \mathrm{mg} / \mathrm{mL}$ PCF/DMF for 45 minutes. (C) Analysis of $1 \mu \mathrm{M}$ DA current produced post- and pre-dip coating $1 \mathrm{mg} / \mathrm{mL}$ PCF/DMF. Analysis shows roughly no increase in oxidation current following $10 \mathrm{~min}$ dip coating (black bar). At increased dip coating times dopamine cannot be detected and increases in background current are observed. Faradaic currents are not quantitated due to noise causing loss of signal; therefore, only changes in capacitive current are shown for dip coating for 20,45, and 60 min. The blue bar represents the background current after 20 min dip-coating, the yellow bar represents the background current changes after $45 \mathrm{~min}$ dip-coating, and the green bar represents the background current changes after $60 \min (n=8)$. 
A

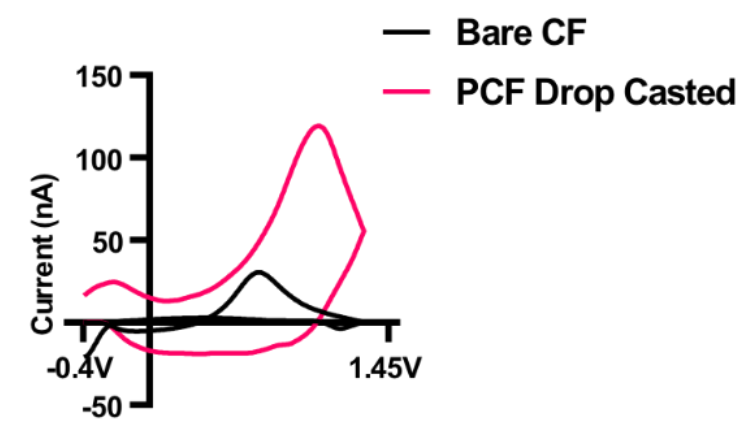

B

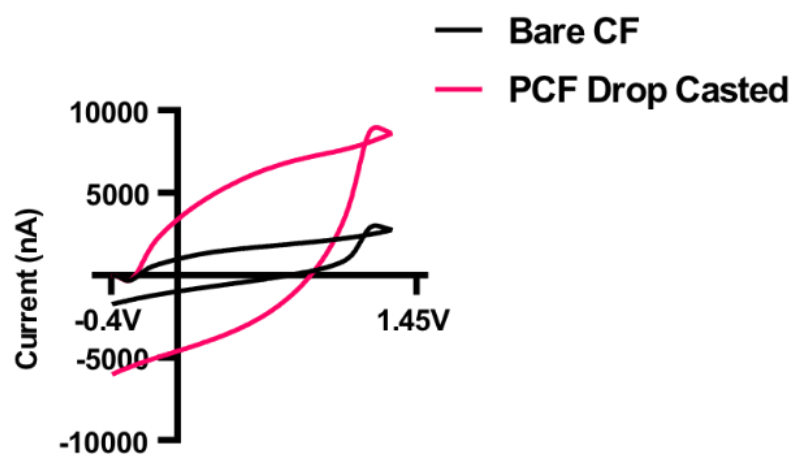

C.
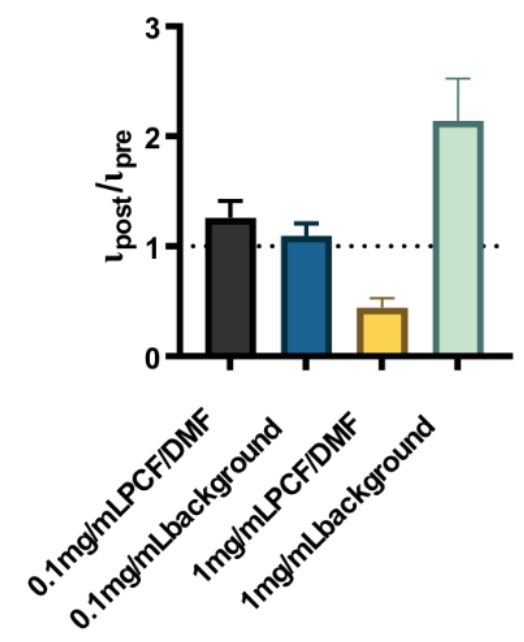

Figure S-4: Modifying CFME with porous carbon nanofibers using drop casting shows varying increases in background current accompanied by unstable changes in oxidation current. $(A)$ Example CV of $1 \mu \mathrm{M}$ DA before and after drop casting of $0.1 \mathrm{mg} / \mathrm{mL}$ PCF/DMF. (B) Example background current CV before and after drop casting of $0.1 \mathrm{mg} / \mathrm{mL}$ PCF/DMF. (C) Analysis of 1 $\mu \mathrm{M}$ DA oxidation current changes after drop casting modification at two different concentrations of PCF in DMF $(n=7)$. Small insignificant changes in DA current and background current were observed with $0.1 \mathrm{mg} / \mathrm{mL}$ PCF drop-casting. A large decrease in current was observed at $1 \mathrm{mg} / \mathrm{mL}$ PCF drop-casting (yellow) accompanied by a major increase background current (green). 


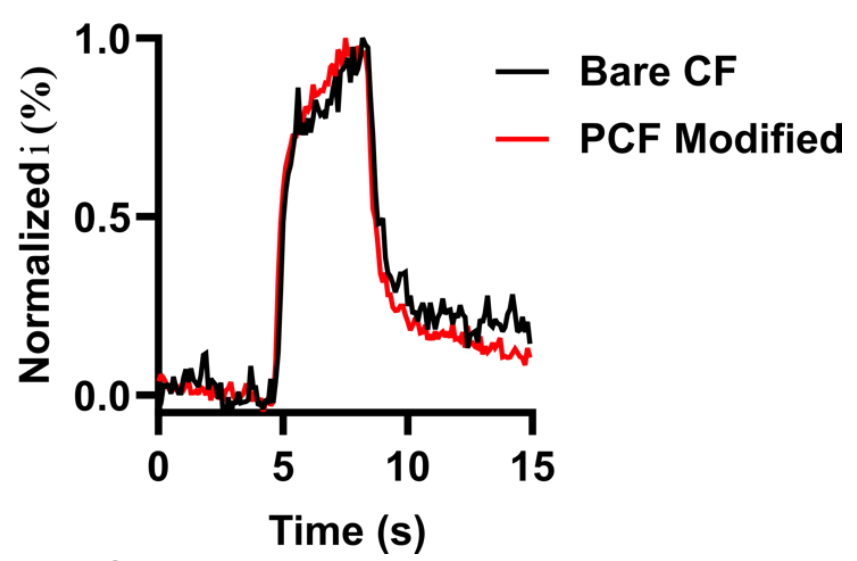

Figure S-5: Example normalized current versus time trace for $1 \mu \mathrm{M}$ DA before (black) and after PCF modification (red). The 10 to $90 \%$ rise significantly decreased after modification ( $2.2 \pm 0.1$ $\mathrm{s}$ to $1.9 \pm 0.1 \mathrm{~s}^{*}$, paired t-test, $\mathrm{p}=0.0425, \mathrm{n}=33$ ). 


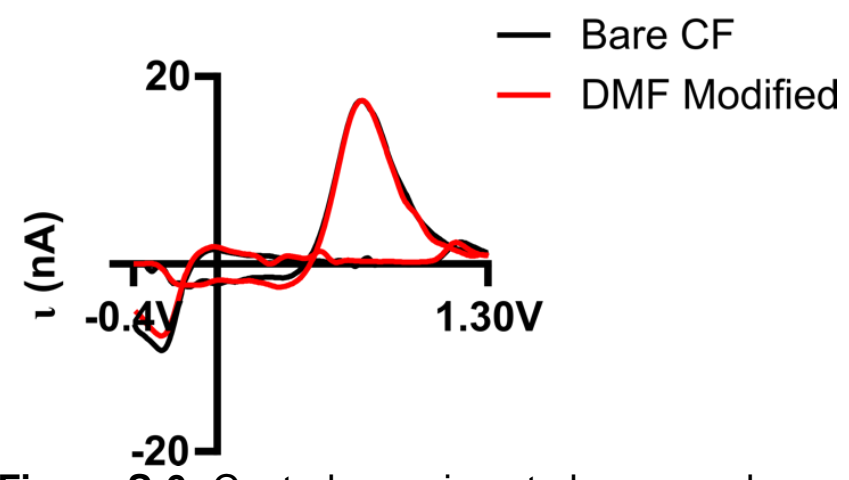

Figure S-6: Control experiment shows no change in DA current after electrodepositing in DMF alone. Example dopamine CVs at bare CFME (black) and modified CMFE (red). This experiment was repeated at 6 electrodes. 


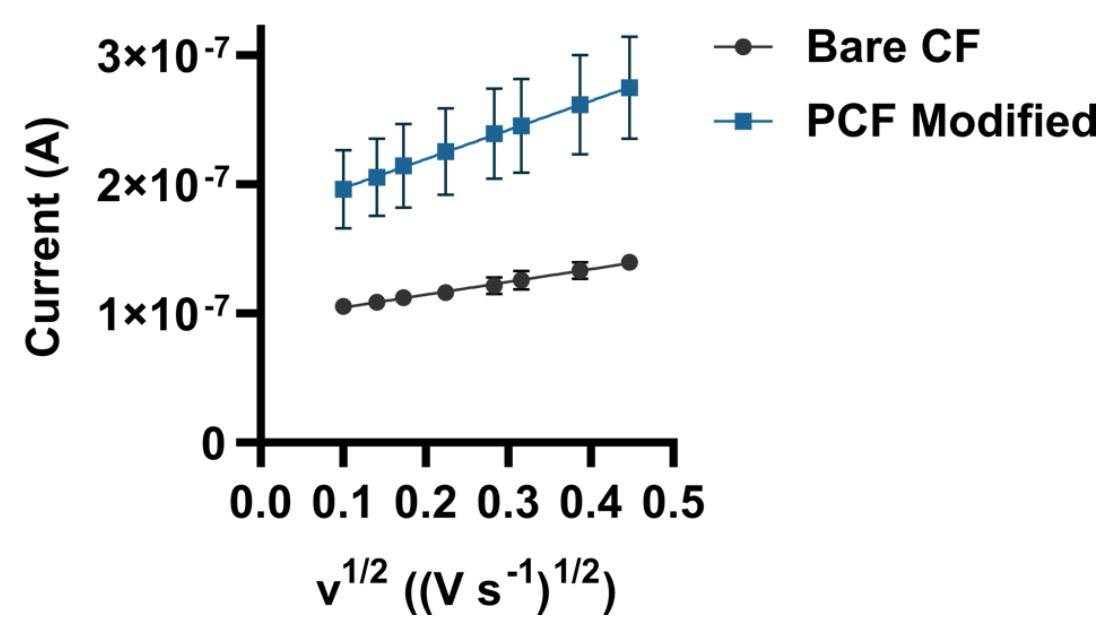

Figure S-7: Oxidative peak current versus square root of scan rate for $\mathrm{Ru}\left(\mathrm{NH}_{3}\right)^{+2 /+3}$ using bare CF and PCF modified CF shows increased electroactive surface area of electrode following modifications. A scan rate investigation was used at scan rates from 10 to $200 \mathrm{mV} \mathrm{s}^{-1}$ and the reversible Randles Sevcik equation was used for electroactive surface area determination ( $n=$ 4). 


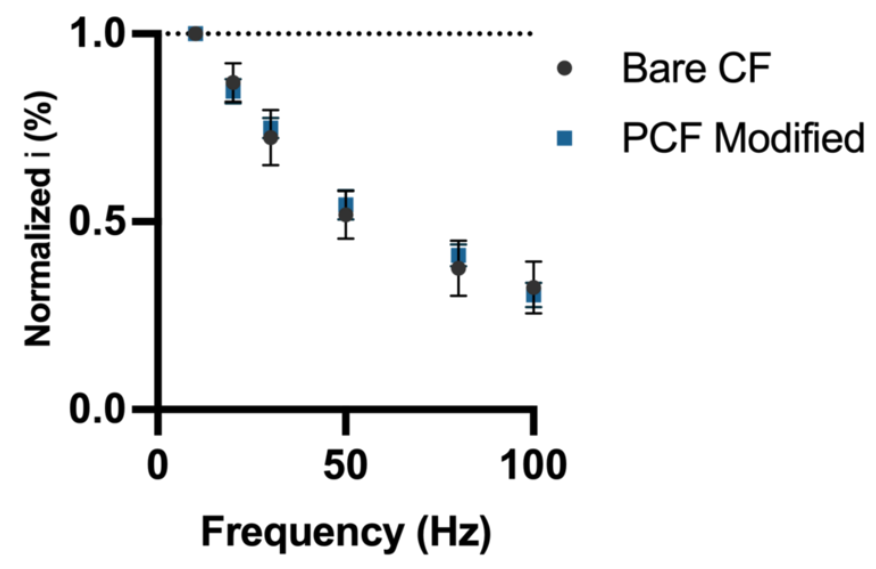

Figure S-8: Current decreases for both bare carbon fiber (Bare CF) and PCF-modified CF as a function of waveform application frequency from 10 to $100 \mathrm{~Hz}$. $(n=6)$ 
Table S1: Comparison of the electrochemical performance of other common carbon material modifications on carbon-fiber microelectrodes.

\begin{tabular}{|c|c|c|c|c|}
\hline Electrode & $\begin{array}{l}\text { Sensitivity } \\
\left(\mathrm{nA} \mu \mathrm{M}^{-1}\right)\end{array}$ & $\begin{array}{l}\text { Limit of } \\
\text { Detection } \\
(\mathrm{nM})\end{array}$ & $\begin{array}{l}\text { Frequency } \\
\text { Independence }\end{array}$ & $\begin{array}{l}\text { Redox } \\
\text { Cycling of DA }\end{array}$ \\
\hline $\begin{array}{l}\text { PCF-CF (this } \\
\text { paper) }\end{array}$ & $12.7 \pm 0.5$ & $3.6 \pm 0.7$ & No & Yes \\
\hline${ }^{7} \mathrm{GO}-\mathrm{CF}$ & $41 \pm 2$ & 11 & Not reported & Not reported \\
\hline $\begin{array}{l}{ }^{8} \text { SWCNT-CF } \\
\text { (Note:Disk } \\
\text { electrodes) }\end{array}$ & $\begin{array}{l}\text { Not } \\
\text { reported }\end{array}$ & $17 \pm 3$ & $\begin{array}{l}\text { Improved } \\
\text { frequency } \\
\text { independence }\end{array}$ & Yes \\
\hline${ }^{9} \mathrm{OX}-\mathrm{CNH}-\mathrm{CF}$ & $\begin{array}{l}\text { Not } \\
\text { reported }\end{array}$ & $6 \pm 2$ & Not reported & Not reported \\
\hline
\end{tabular}




\section{Supporting References}

(1) Zhou, Z.; Liu, T.; Khan, A. U.; Liu, G. Controlling the Physical and Electrochemical Properties of Block Copolymer-Based Porous Carbon Fibers by Pyrolysis Temperature. Mol. Syst. Des. Eng. 2020, 5 (1), 153-165. https://doi.org/10.1039/C9ME00066F.

(2) Zhou, Z.; Liu, T.; Khan, A. U.; Liu, G. Block Copolymer-Based Porous Carbon Fibers. Sci. Adv. 2019, 5 (2), eaau6852. https://doi.org/10.1126/sciadv.aau6852.

(3) Composition Design of Block Copolymers for Porous Carbon Fibers / Chemistry of Materials https://pubs.acs.org/doi/abs/10.1021/acs.chemmater.9b02918 (accessed 2021 -03 -31).

(4) Serrano, J. M.; Khan, A. U.; Liu, T.; Xu, Z.; Esker, A. R.; Liu, G. Capacitive Organic Dye Removal by Block Copolymer Based Porous Carbon Fibers. Adv. Mater. Interfaces 2020, 7 (16), 2000507. https://doi.org/10.1002/admi.202000507.

(5) Armatas, G. S.; Petrakis, D. E.; Pomonis, P. J. A Method of Distinction between Microporosity and Mesoporosity Using BET-Scatchard Plots. Microporous Mesoporous Mater. 2005, 83 (1), 251-261. https://doi.org/10.1016/j.micromeso.2005.05.005.

(6) Li, C.; Li, Q.; Kaneti, Y. V.; Hou, D.; Yamauchi, Y.; Mai, Y. Self-Assembly of Block Copolymers towards Mesoporous Materials for Energy Storage and Conversion Systems. Chem. Soc. Rev. 2020, 49 (14), 4681-4736. https://doi.org/10.1039/D0CS00021C.

(7) Chang, Y.; Venton, B. J. Optimization of Graphene Oxide-Modified Carbon-Fiber Microelectrode for Dopamine Detection. Anal. Methods 2020, 12 (22), 2893-2902. https://doi.org/10.1039/D0AY00310G.

(8) Xiao, N.; Venton, B. J. Rapid, Sensitive Detection of Neurotransmitters at Microelectrodes Modified with Self-Assembled SWCNT Forests. Anal. Chem. 2012, 84 (18), 7816-7822. https://doi.org/10.1021/ac301445w.

(9) Puthongkham, P.; Yang, C.; Venton, B. J. Carbon Nanohorn-Modified Carbon Fiber Microelectrodes for Dopamine Detection. Electroanalysis 2018, 30 (6), 1073-1081. https://doi.org/10.1002/elan.201700667. 